\title{
Commentary \\ Recently published papers: Novel therapies in chronic obstructive pulmonary disease, cardiac chemicals and intensive care outcomes
}

\author{
Uma M Bandarupalli and Gareth D Williams
}

Adult Critical Care Unit, Leicester Royal Infirmary, Leicester LE1 5WW, UK

Corresponding author: Gareth D Williams, gareth.williams@uhl-tr.nhs.uk

Critical Care 2009, 13:198 (doi:10.1186/cc8130)

This article is online at http://ccforum.com/content/13/5/198

(c) 2009 BioMed Central Ltd

\begin{abstract}
The burden of chronic obstructive pulmonary disease on intensive care is heavy, but newer modalities of treatment are now available to improve outcomes. Cardiac-based research continues to generate new drugs and tests to better outcome and aid in early diagnosis. And how do various intensive care interventions compare in improving clinical and functional outcomes?
\end{abstract}

\section{Novel therapies in chronic obstructive pulmonary disease}

Chronic obstructive pulmonary disease (COPD) continues to be a major medical problem. With no curative therapy being available, management of severe exacerbations of COPD should be directed at relieving symptoms and restoring functional capacity [1]. Mortality from COPD continues to rise and the World Health Organisation predicts it will be the third leading cause of death by 2030 [2]. There is current evidence suggesting that quality improvement interventions ought to be focussed at recognition of complications and treatment of the same [3].

We examine a randomized double-blind, cross-over study published by Israeli investigators Staz and Rav [4] looking at the effect of $\mathrm{N}$-acetylcysteine (NAC) on airflow limitation in COPD. Twenty-four moderate to severe COPD patients were randomized to placebo or nebulised NAC $(1,200 \mathrm{mg}$ per day) treatment twice daily for 6 weeks. This was followed by a 2-week washout period and patients were crossed over to alternative therapy for a further 6 weeks. Evaluation of forced vital capacity, inspiratory capacity and other parameters was made after each 6-week treatment. The results showed that forced vital capacity and inspiratory capacity were higher, especially after exercise, in the NAC group compared with placebo. Furthermore, endurance time was longer after NAC treatment compared with placebo treatment. They concluded that use of NAC in moderate to severe COPD patients produces a beneficial effect on physical performance, probably due to reduction in dynamic hyperinflation with a relatively short course of treatment. It is impossible to extrapolate these data to the ICU population, but this paper may ignite renewed interest in NAC therapy, possibly reducing the need for invasive ventilation or the number of ventilator days. Only further studies will tell.

Roflumilast is a new phosphodiesterase- 4 inhibitor, and may offer some hope for patients with moderate to severe COPD. The Lancet published two randomised clinical trials suggesting that this new agent decreases COPD exacerbations and the need for adjuvant corticosteroid therapy, improves lung function tests and reported functional capacity when compared with placebo $[5,6]$. It seems likely that roflumilast could become an important concomitant therapy in certain COPD subsets.

\section{Cardiac chemicals}

Acute myocardial infarction is often a diagnostic challenge in the critically ill. A recent muticentre, emergency-room-based study by Reichlin and colleagues [7] looked at the difference between sensitive troponin assays and standard troponin assays. Cardiac troponin levels were determined in a blinded fashion with the use of four sensitive assays and a standard assay. The final diagnosis was adjudicated by two independent cardiologists. Acute myocardial infarction was diagnosed in $17 \%$ of cases and accuracy was ascertained using AUC (area under the receiver operating characteristic curve). The sensitive assays had significant 20 to $25 \%$ increased sensitivity compared to standard assays. The authors concluded that this new diagnostic assay should substantially improve the early detection of acute myocardial infarction and, therefore, outcome.

Atrial fibrillation is the direct cause of one in four strokes in the over 80 year olds. Treating individuals with warfarin 
reduces the rate of stroke for those who have atrial fibrillation by approximately one-half to two- thirds [8]. But the increased risk of haemorrahge and need for monitoring has sparked much research, and a recent study tested a new direct thrombin inhibitor, dabigatran, against warfarin [9]. Connolly and colleagues [9] employed a non-inferiority trial, randomly assigning 18,113 patients with atrial fibrillation and a risk of stroke to receive, in a blinded fashion, fixed doses of dabigatran (110 mg or $150 \mathrm{mg}$ twice daily) or, in an unblinded fashion, adjusted-dose warfarin. The median duration of the follow-up period was 2 years. The primary outcome was stroke or systemic embolism. The study boasts of a favourable outcome towards dabigatran in terms of both preventing stroke/systemic embolism and lesser rates of major haemorrhage.

\section{Intensive care outcomes}

Burtin and colleagues [10] investigated whether a daily exercise session, using a bedside cycle ergometer, is a safe and effective intervention in preventing or attenuating the decrease in functional exercise capacity that is associated with prolonged ICU stay. Ninety critically ill patients with expected prolonged stay were randomized to bedside ergometer along with standardized therpay (physiotherapy). Quadriceps force and functional status were assessed at ICU discharge and hospital discharge. Six-minute walking distance was measured at hospital discharge. There was no adverse effect from the outcome among survivors and the intervention group had significant functional improvement at hospital discharge.

Another investigating party looked at the gender impact on the outcomes of critically ill patients with nosocomial infections [11]. It was a retrospective study based in an 18bed general ICU. Multivariable logistic regression analysis was used to control for confounders in the evaluation of gender impact on ICU death post-nosocomial infections. Over 5,000 patient admissions were studied and the conclusion was that female ICU patients developing nosocomial infections seem to be at increased risk of ICU mortality, after carefully controlling for other prognostic factors. Further studies are needed to elucidate the pathophysiology underlying this gender-related difference, to devise tailored gender-specific therapies that might improve outcomes.

Alberda and colleagues [12] published a multicentre observational study examining the relationship between the amount of energy and protein administered during ICU stay and clinical outcomes in critically ill patients, and the extent to which pre-morbid nutritional status influenced this relationship. The type and amount of nutrition received were recorded daily for a maximum of 12 days. Patients were followed prospectively to determine 60-day mortality and ventilator-free days. Data were collected on 2,772 mechanically ventilated patients who received an average of $1,034 \mathrm{kcal} /$ day and $47 \mathrm{~g}$ protein/day. An increase of
$1,000 \mathrm{cal} /$ day was associated with reduced mortality. The effect of increased calories associated with lower mortality was observed in patients with a body mass index $<25$ and $\geq 35$ with no benefit for patients with a body mass index of 25 to $<35$. Similar results were observed when comparing increased protein intake and its effect on mortality. Perhaps this is an intervention ICUs would want to seriously consider.

Acute kidney injury (AKI) has been the subject of NCEPOD (National Confidential Enquiry into Patient Outcome and Death) interest recently and data into provision of care and outcomes have been published [13]. French investigators Floccard and colleagues [14] have looked into 6-month outcomes from $\mathrm{AKI}$ requiring renal replacement therapy (RRT) in the ICU. AKI was defined by the RIFLE score. Quality of life was assessed using the Short Form Health Survey (SF-36) questionnaire. The results indicated that patients who received RRT for AKI had a high mortality rate 6 months after RRT finished. Survivors (38\%) at follow-up had a lower level of quality of life compared to a reference population, and their physical health was more affected than their mental health. Most of the patients were independent in daily activities and returned home. Twelve percent of survivors were still receiving RRT at follow-up.

Acute necrotising pancreatitis is a serious condition with a high mortality rate [15]. A recent prospective study [16] assessed quality of life outcomes in 31 patients. Survivors (68\%) were assessed at 3, 6 and 12 months following hospital discharge by an investigator blinded to their previous treatment. Health related quality of life was assessed by the Short Form Health Survey (SF-36) questionnaire and functional outcome by the six-minute walk test. They concluded that, in the first year after the insult, patients showed improvement in their physical components of quality of life and in their physical function, but their outcome at 12 months was still poor compared to the general population. This patient group in particular may benefit from a structured rehabilitation programme continuing after hospital discharge.

\section{Competing interests}

The authors declare that they have no competing interests.

\section{References}

1. Standards for the diagnosis and care of patients with chronic obstructive pulmonary disease. American Thoracic Society. Am J Respir Crit Care Med 1995, 152:S77-121.

2. World Health Organization Statistics: COPD predicted to be third leading cause of death in 2030 [http://www.who.int/ gard/news_events/World_Health_Statistics_2008/en/index.html]

3. Zvezdin B, Milutinov S, Kojicic M, Hadnadjev M, Hromis S, Markovic M, Gajic O: A postmortem analysis of major causes of early death in patients hospitalized with COPD exacerbation. Chest 2009, 136:376-380.

4. Stav D, Raz M: Effect of $\mathrm{N}$-acetylcysteine on air trapping in COPD: a randomized placebo-controlled study. Chest 2009, 136:381-386.

5. Calverley PMA, Rabe KF, Goehring U-M, Kristiansen S, Fabbri LM, Martinez FJ; for the M2-124 and M2-125 Study Groups: Roflumilast in symptomatic chronic obstructive pulmonary disease: two randomised clinical trials. Lancet 2009, 374:685-694. 
6. Fabbri LM, Calverley PMA, Izquierdo-Alonso JL, Bundschuh DS, Brose M, Martinez FJ, Rabe KF; for the M2-127 and M2-128 Study Groups: Roflumilast in moderate-to-severe chronic obstructive pulmonary disease treated with longacting bronchodilators: two randomised clinical trials. Lancet 2009, 374: 695-703.

7. Reichlin T, Hochholzer W, Bassetti S, Steuer S, Stelzig C, Hartwiger S, Biedert S, Schaub N, Buerge C, Potocki M, Noveanu M, Breidthardt T, Twerenbold R, Winkler K, Bingisser R, Mueller $\mathrm{C}$ : Early diagnosis of myocardial infarction with sensitive cardiac troponin assays. N Engl J Med 2009, 361:858-867.

8. National Institute of Neurological Disorders and Stroke: Atrial Fibrillation and Stroke Information Page [http://www.ninds.nih. gov/disorders/atrial_fibrillation_and_stroke/atrial_fibrillation_and_ stroke.htm]

9. Connolly SJ, Ezekowitz MD, Yusuf S, Eikelboom J, Oldgren J, Parekh A, Pogue J, Reilly PA, Themeles E, Varrone J, Wang S, Alings M, Xavier D, Zhu J, Diaz R, Lewis BS, Darius H, Diener HC, Joyner CD, Wallentin L; RE-LY Steering Committee and Investigators: Dabigatran versus warfarin in patients with atrial fibrillation. N Engl J Med 2009, 361:1139-1151.

10. Burtin $C$, Clerckx B, Robbeets $C$, Ferdinande $P$, Langer D, Troosters T, Hermans G, Decramer M, Gosselink R: Early exercise in critically ill patients enhances short-term functional recovery. Crit Care Med 379:2499-2505.

11. Combes A, Luyt CE, Trouillet JL, Nieszkowska A, Chastre J: Gender impact on the outcomes of critically ill patients with nosocomial infections. Crit Care Med. 379:2506-2511.

12. Alberda C, Gramlich L, Jones N, Jeejeebhoy K, Day AG, Dhaliwal R, Heyland DK: The relationship between nutritional intake and clinical outcomes in critically ill patients: results of an international multicenter observational study. Intensive Care Med 2009, 35:1728-1737.

13. National Confidential Enquiry into Patient Outcome and Death: Adding Insult to Injury - a Review of the Care of Patients Who Died in Hospital with a Primary Diagnosis of Acute Kidney Injury (Acute Renal Failure) [http://www.ncepod. org.uk/2009report1/Downloads/AKI_report.pdf]

14. Delannoy B, Floccard B, Thiolliere F, Kaaki M, Badet M, Rosselli S, Ber CE, Saez A, Flandreau G, Guérin C: Six-month outcome in acute kidney injury requiring renal replacement therapy in the ICU: a multicentre prospective study. Intensive Care Med 2009 , in press.

15. Whitcomb DC: Clinical practice: acute pancreatitis. $N$ Engl J Med 2006, 354:2142-2150.

16. Wright SE, Lochan R, Imrie K, Baker C, Nesbitt ID, Kilner AJ, Charnley RM: Quality of life and functional outcome at 3,6 and 12 months after acute necrotising pancreatitis. Intensive Care Med 2009, in press. 